\title{
Influencia de la familia como factor protector de conductas de riesgo en escolares chilenos
}

\author{
Sylvia Santander $R^{1}$, Tamara Zubarew $\mathbf{G}^{2}$, Lucía Santelices $C^{3}$, \\ Pamela Argollo M²a, Jaime Cerda L4, Mariana Bórquez P1. \\ Family influence as a protective \\ factor against risk behaviors in \\ Chilean adolescents
}

Background: Family is an important protective factor in the prevention of risk behaviors in Chilean adolescents. Aim: To determine the prevalence of risk and protective factors and behaviors in Chilean students, and to study the association between family functioning and adolescent risk behaviors. Material and methods: Descriptive cross sectional study of 815 adolescents aged $15 \pm 2$ years ( $51 \%$ male), studying $7^{\text {th }}$ to $12^{\text {th }}$ grade in three educational facilities in Santiago, Chile. They answered the Adolescent Risk Behavior Score, adapted from the Adolescent Health Survey and the "How is your family" survey of the Pan American Health Organization. Results: Tobacco, alcohol and marihuana consumption was reported by 31\%, 27\% and $24 \%$ of students, respectively. Twelve percent reported being sad all the time, $23 \%$ being desperate and $13 \%$ being anxious. Forty four percent have had a sexual intercourse. The risk to have these behaviors was greater in adolescents who perceive their families as dysfunctional. Conclusions: In this sample, there was a high prevalence of risk behaviors, emotional symptoms and premature sexual intercourse. The risk of having these behaviors is higher among students who perceive their families as dysfunctional. The study emphasizes the protective function of family in the prevention of risk behaviors in Chilean students (Rev Méd Chile 2008; 136: 317-24).

(Key words: Adolescent; Risk-taking; Sexual behavior)

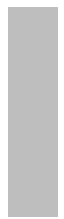

Recibido el 8 de marzo, 2007. Aceptado el 2 de octubre, 2007.

${ }^{1}$ Dirección de Investigación, Universidad Metropolitana de Ciencias de la Educación. Departamentos de ${ }^{2}$ Pediatría y de Salud Pública ${ }^{4}$; Facultad de Medicina. ${ }^{3}$ Subdirección de Investigación y Docencia, Facultad de Educación. Pontificia Universidad Católica de Chile. Santiago de Chile.

${ }^{a}$ Becada de Pediatría, Pontificia Universidad Católica de Chile

$\mathrm{D}$ urante las últimas décadas, los procesos de transición demográfica y epidemiológica que han experimentado diversos países, junto a impor-

Correspondencia a: Dra. Tamara Zubarew G. Departamento de Pediatría, Facultad de Medicina, Pontificia Universidad Católica de Chile. Lira 85, Santiago, Chile. C.P.833-0074. Tel: 354 3402. Fax: 638 4307. E mail: tzubarew@med.puc.cl tantes cambios socioculturales, han cambiado el perfil de morbilidad y mortalidad de los adolescentes. Hoy en día, la mayoría de sus problemas de salud son consecuencia de conductas de riesgo (e.g. abuso de sustancias), trayendo como resultante enfermedades de transmisión sexual, accidentes de tránsito, depresión y suicidio ${ }^{1,2}$. Los cambios descritos han ocurrido en forma paralela 
a cambios en la estructura familiar tales como aumento de los hogares monoparentales y del número de mujeres que han ingresado en la fuerza laboral, volviendo más vulnerable un importante núcleo protector del adolescente. Lo anterior aparece como relevante, porque en el desarrollo de un adolescente, el contexto social en que se desenvuelve -familia y escuela- son los contextos más influyentes. $\mathrm{Al}$ respecto, se ha demostrado que un adecuado funcionamiento familiar es relevante, siendo la disfunción familiar un factor que se asocia a mayor prevalencia de problemas de salud mental en adolescentes, lo que se asocia también con alteración del rendimiento académico, mayor deserción escolar y aumento de la violencia ${ }^{1-7}$.

Lo anterior cobra particular importancia porque la adolescencia es un período de transición en el cual se concretan importantes hitos del desarrollo. La consolidación de la identidad personal, sexual, vocacional, la independencia económica y la conformación de las bases para una posterior relación de pareja estable, objetivos frente a los cuales, el contexto vital del adolescente -familia, escuela, amistades- son un apoyo fundamental. $\mathrm{Al}$ respecto, en la literatura especializada se señala que estos tres contextos actúan como factores protectores, sin embargo, en ciertas circunstancias pueden ser un obstáculo, actuando más bien como factores de riesgo ${ }^{8-11}$.

Frente a lo anterior, este trabajo se propuso dos objetivos, determinar la prevalencia de factores y conductas tanto protectoras como de riesgo en adolescentes chilenos, y estudiar la correlación entre la percepción del funcionamiento familiar del adolescente, sus síntomas emocionales y conductas de riesgo.

\section{MATERIAL Y MÉTODO}

Sujetos de estudio. Estudio descriptivo transversal realizado entre abril y junio de 2006, en 815 adolescentes entre $7^{\circ}$ básico y $4^{\circ}$ medio, provenientes de tres establecimientos educacionales de la ciudad de Santiago. Se utilizó la clasificación de Adimark para determinar nivel socioeconómico (NSE), que considera escolaridad y profesión de los padres. Se eligió para el estudio un colegio particular de NSE alto, un colegio particular subvencionado de NSE medio y un colegio municipalizado, de NSE bajo. La participación de los adolescentes en el estudio fue voluntaria, exigiéndose consentimiento informado.

Instrumento de evaluación. Cuestionario ECRA (Escala de Conductas de Riesgo en Adolescentes), autoadministrado y confidencial, adaptado del cuestionario "Adolescent Health Survey"3 y validado en Chile ${ }^{12}$, consistente en 70 preguntas a responder en una hora durante la jornada regular de clases. Las variables incluidas fueron: demográficas, constitución y funcionamiento familiar, situación escolar y laboral de los padres, antecedentes personales y familiares de salud, sexualidad y salud reproductiva, hábitos personales (e.g. consumo de sustancias, actividad física), conductas de riesgo (e.g. violencia física o emocional) y existencia de redes de apoyo. Dirigidamente se interrogó por consumo de sustancias, conductas sexuales, síntomas emocionales y dinámica de comunicación familiar. Al cuestionario se agregó la encuesta "¿Cómo es tu familia?" desarrollada por la Organización Panamericana de la Salud $^{13}$. A pesar que el instrumento original se aplica a varios miembros de la familia, en este estudio se aplicó solamente a los adolescentes y exploró su percepción acerca de diversas dimensiones de la vida familiar, tales como funcionamiento, comunicación, cohesión y dinámica familiar.

Estadística. Los datos registrados fueron tabulados según su distribución de frecuencias. Se calculó prevalencia de cuatro grupos de variables dependientes (consumo de sustancias, síntomas emocionales, dinámica de comunicación familiar y actividad sexual). Posteriormente, se calculó la asociación entre percepciones de disfunción familiar (definida operacionalmente a partir de las percepciones de los estudiantes en función de ausencia de comunicación o cohesión entre los miembros de la familia, ausencia de tiempo para compartir en familia, ausencia de recursos o fuentes de apoyo ante la presencia de dificultades y una mala organización familiar) y las variables dependientes (con excepción de variables del grupo "dinámicas de comunicación familiar") mediante la prueba de chi cuadrado $\left(\chi^{2}\right)$, expresándose la magnitud del riesgo en términos de odds ratio (OR). Se calculó el riesgo de consumo de 
sustancias entre adolescentes según el patrón de consumo de sustancias de su padre y madre $\left(\chi^{2}\right.$ y OR). Se construyeron intervalos de confianza a 95\%, considerándose significativo un $\alpha$ menor a 0,05. Los datos fueron tabulados y analizados utilizando el software estadístico SPSS14 ${ }^{\circledR}$.

\section{Resultados}

Un total de 815 adolescentes aceptó responder la encuesta; $56,3 \%$ provenientes del colegio de NSE bajo, $27,4 \%$ del colegio de NSE alto y $16,3 \%$ del colegio de NSE medio. El 50,6\% de los estudiantes corresponden a varones, siendo la edad promedio $15,1 \pm 1,7$ años (rango 10-20). El 14,5\% cursaba enseñanza básica $\left(7^{\circ}\right.$ y $8^{\circ}$ básico) y $85,5 \%$ enseñanza media $\left(1^{\circ}\right.$ a $4^{\circ}$ medio $)$.

Consumo de sustancias. La Tabla 1 resume las prevalencias y OR de consumo de sustancias, según sexo. Considerando ambos sexos, la tasa más alta corresponde al consumo de tabaco $(36,0 \%)$, seguida por el consumo de alcohol (26,6\%) y marihuana $(24,2 \%)$. La prevalencia de consumo de tabaco y alcohol según rangos de edad (menor de 13 años, 13-16 años y mayores de 16 años) fue más alta a edades mayores, siendo para tabaco $13,8 \%, 32,2 \%$ y $50,2 \%$ y para alcohol $10,0 \%, 25,8 \%$ y $35,7 \%$, respectivamente. La Tabla 2 resume el OR de consumo de sustancias, según percepción del funcionamiento familiar. Los escolares integrantes de hogares percibidos por los estudiantes como disfuncionales tuvieron riesgo significativamente mayor de consumo de tabaco, marihuana, tranquilizantes, alcohol y cocaína, sin embargo, los dos últimos OR no son estadísticamente significativos.

La Tabla 3 resume el OR de consumo de sustancias del adolescente según el consumo de igual sustancia por parte del padre o madre. Los adolescentes cuyos padres consumían tabaco, alcohol o marihuana tenían significativamente más riesgo de consumir dichas sustancias en comparación a los adolescentes cuyos padres no las consumían; similar situación ocurre en el caso de las madres consumidoras.

Tabla 1. Prevalencia (\%) y $0 \mathrm{R}$ de consumo de sustancias, síntomas emocionales y actividad sexual, según sexo

\begin{tabular}{|c|c|c|c|c|c|}
\hline & Varones & Mujeres & OR & IC 95\% & $\mathrm{p}$ \\
\hline \multicolumn{6}{|l|}{ Sustancia } \\
\hline Tabaco ${ }^{\mathrm{a}}$ & 31,6 & 40,5 & 0,68 & $0,50-0,92$ & $<0,01$ \\
\hline Alcohol $^{\mathrm{a}}$ & 30,7 & 22,5 & 1,53 & $1,10-2,12$ & $<0,01$ \\
\hline Marihuana ${ }^{\mathrm{b}}$ & 26,3 & 22,1 & 1,26 & $0,90-1,78$ & ns \\
\hline Tranquilizantes ${ }^{\mathrm{b}}$ & 8,8 & 16,3 & 0,50 & $0,31-0,78$ & $<0,01$ \\
\hline Cocaína ${ }^{b}$ & 5,8 & 4,1 & 1,45 & $0,72-2,93$ & ns \\
\hline \multicolumn{6}{|l|}{ Síntoma emocional } \\
\hline Inestabilidad ${ }^{\mathrm{C}}$ & 62,0 & 79,8 & 0,41 & $0,30-0,58$ & $<0,001$ \\
\hline Desesperado $^{\mathrm{d}}$ & 19,8 & 25,6 & 0,72 & $0,51-1,02$ & ns \\
\hline Autocontrol $^{\mathrm{e}}$ & 17,2 & 17,1 & 1,00 & $0,68-1,48$ & ns \\
\hline Tristeza $^{\mathrm{f}}$ & 6,4 & 19,4 & 0,28 & $0,17-0,46$ & $<0,001$ \\
\hline Ansiedadg & 9,7 & 16,8 & 0,53 & $0,34-0,84$ & $<0,01$ \\
\hline \multicolumn{6}{|l|}{ Actividad sexual } \\
\hline Relaciones $^{\mathrm{h}}$ & 47,8 & 39,1 & 1,42 & $1,06-1,91$ & $<0,02$ \\
\hline
\end{tabular}

$\mathrm{OR}=$ odds ratio varones vs mujeres. $\mathrm{ns}=$ no significativo.

${ }^{a}$ Consumo mensual, semanal o diario. ${ }^{b}$ Consumo alguna vez. ${ }^{c}$ Inestable e inseguro todo el tiempo. ${ }^{d}$ Desesperado, descorazonado, siente que nada vale la pena; bastante o en extremo. ${ }^{\text {eIncapaz de }}$ controlar la conducta todo el tiempo. ${ }^{\mathrm{f}}$ Triste todo el tiempo. ${ }^{\mathrm{g}}$ Ansioso y preocupado todo el tiempo.

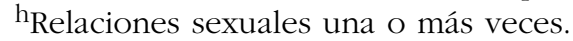




\section{Tabla 2. Prevalencia (\%) y O R de consumo de sustancias y síntomas emocionales, según funcionalidad del hogar}

\begin{tabular}{|c|c|c|c|c|c|}
\hline & $\mathrm{DF}(+)$ & DF (-) & OR & IC 95\% & $\mathrm{p}$ \\
\hline \multicolumn{6}{|l|}{ Sustancias } \\
\hline Tabaco ${ }^{\mathrm{a}}$ & 38,7 & 30,2 & 1,46 & $1,03-2,07$ & 0,03 \\
\hline Alcohol $^{\mathrm{a}}$ & 29,3 & 24,2 & 1,30 & $0,89-1,89$ & ns \\
\hline Marihuana $^{\mathrm{b}}$ & 27,2 & 17,5 & 1,76 & $1,17-2,66$ & $<0,01$ \\
\hline Tranquilizantes ${ }^{\mathrm{b}}$ & 16,1 & 8,1 & 2,19 & $1,26-3,86$ & $<0,01$ \\
\hline Cocaína ${ }^{b}$ & 6,4 & 3,0 & 2,23 & $0,92-5,66$ & ns \\
\hline \multicolumn{6}{|l|}{ Sentimientos } \\
\hline Inestabilidad ${ }^{\mathrm{C}}$ & 77,1 & 64,1 & 1,89 & $1,32-2,69$ & $<0,001$ \\
\hline Desesperado $^{d}$ & 28,1 & 13,9 & 2,42 & $1,56-3,75$ & $<0,001$ \\
\hline Autocontrole & 21,2 & 10,7 & 2,25 & $1,37-3,70$ & $<0,001$ \\
\hline Tristeza $^{\mathrm{f}}$ & 16,3 & 7,6 & 2,36 & $1,34-4,19$ & $<0,01$ \\
\hline Ansiedadg & 16,3 & 8,9 & 2,00 & $1,17-3,44$ & $<0,01$ \\
\hline \multicolumn{6}{|l|}{ Actividad sexual } \\
\hline Relaciones $^{\mathrm{h}}$ & 48,0 & 34,6 & 1,74 & $1,24-2,44$ & $<0,001$ \\
\hline
\end{tabular}

$\mathrm{DF}=$ disfunción familiar. $\mathrm{OR}=$ odds ratio disfunción familiar (+) vs disfunción familiar (-). ns = no significativo. ${ }^{\mathrm{a} C}$ Consumo mensual, semanal o diario. ${ }^{\mathrm{b}}$ Consumo alguna vez. ${ }^{\mathrm{C}}$ Inestable e inseguro todo el tiempo. ${ }^{\mathrm{d}}$ Desesperado, descorazonado, siente que nada vale la pena; bastante o en extremo. ${ }^{\mathrm{I}}$ Incapaz de controlar conducta todo el tiempo. ${ }^{\mathrm{f}}$ Triste todo el tiempo. ${ }^{\mathrm{g}}$ Ansioso y preocupado todo el tiempo. ${ }^{h}$ Relaciones sexuales una o más veces.

Tabla 3. Prevalencia (\%) y OR de consumo de sustancias entre los hijos, según consumo de la misma sustancia por el padre y la madre

\begin{tabular}{|lccccc|}
\hline Consumo hijo(a) & Padre (+) & Padre (-) & OR ${ }^{\text {a }}$ & IC 95\% & p \\
\hline Tabaco $^{\text {a }}$ & 47,0 & 27,3 & 2,37 & $1,73-3,25$ & $<0,001$ \\
Alcohol $^{\text {a }}$ & 43,3 & 16,3 & 3,92 & $2,75-5,58$ & $<0,001$ \\
Marihuana $^{\text {b }}$ & 55,9 & 20,7 & 4,86 & $2,72-8,70$ & $<0,001$ \\
Consumo hijo(a) & Madre (+) & Madre (-) & OR b & IC 95\% & p \\
\hline Tabaco $^{\text {a }}$ & 46,2 & 28,6 & 2,14 & $1,58-2,90$ & $<0,001$ \\
Alcohol $^{\text {a }}$ & 53,5 & 21,9 & 4,11 & $2,73-6,20$ & $<0,001$ \\
Marihuana $^{\text {b }}$ & 64,7 & 23,1 & 6,09 & $2,05-18,76$ & $<0,001$ \\
\hline
\end{tabular}

$\mathrm{OR}^{\mathrm{a}}=$ odds ratio padre $(+)$ vs padre $(-) . \mathrm{OR}^{\mathrm{b}}=$ odds ratio madre $(+)$ vs madre $(-)$. ns $=$ no significativo. ${ }^{\mathrm{a} C}$ Consumo mensual, semanal o diario. ${ }^{\mathrm{b}}$ Consumo alguna vez.

Síntomas emocionales. La Tabla 1 resume las prevalencias y OR de síntomas emocionales, según sexo. En conjunto, 12,9\% de los escolares refirió sentirse "triste todo el tiempo", 22,7\% "bastante o extremadamente desesperado, descorazonado y sintiendo que nada vale la pena", $70,9 \%$ "estable y seguro algunas veces o nunca" y $13,2 \%$ "ansioso y preocupado todo el tiempo". Por 
su parte, $17,2 \%$ de los escolares señalaron "no poder controlar su conducta y emociones" todo el tiempo. La Tabla 2 resume el OR de síntomas emocionales, según percepciones de los estudiantes respecto del funcionamiento familiar. El riesgo de presentar la totalidad de las condiciones descritas anteriormente fue significativamente mayor en adolescentes que percibían sus hogares como disfuncionales.

Comunicación familiar. El 48,4\% de los adolescentes señaló que su padre dedica muy rara vez o nunca tiempo para conversar con él, siendo la conversación satisfactoria "frecuentemente o casi siempre" en 34,4\%. Por su parte, $25,6 \%$ de los adolescentes señaló que su madre dedica muy rara vez o nunca tiempo para conversar con ellos, siendo la conversación satisfactoria "frecuentemente o casi siempre" en 53,6\%. El $58,4 \%$ de los adolescentes ha sentido "con poca intensidad o nunca" que hay reglas poco claras en su casa y $12,4 \%$ "sienten con mucha intensidad" que sus padres les dan demasiada libertad. Finalmente, 62,5\% de los adolescentes compartían con frecuencia o casi siempre algunas comidas junto a su familia y $45,7 \%$ realizaban con frecuencia o casi siempre alguna actividad en conjunto.

Conducta sexual. El 43,5\% de los adolescentes declara haber tenido relaciones sexuales, siendo la prevalencia significativamente mayor en hombres que en mujeres (Tabla 1). Analizando ambos sexos en conjunto, esta prevalencia se asoció en forma directa y significativamente a la edad, alcanzando $14,3 \%$ en menores de 13 años, 40,3\% entre 13-16 años y 60,8\% en mayores de 16 años. $\mathrm{Al}$ analizar el grupo de escolares de 14 a 15 años, $55 \%$ ya ha iniciado actividad sexual. La Tabla 2 resume el OR de actividad sexual, según percepción de los estudiantes respecto del funcionamiento familiar. El riesgo de haber tenido relaciones sexuales fue significativamente mayor en adolescentes de hogares percibidos como disfuncionales en comparación a hogares percibidos como funcionales.

\section{DisCUSIÓN}

En estas últimas décadas se ha producido un cambio en el perfil de morbimortalidad de adolescentes, cambios socioculturales y cambios en la familia del adolescente. Es así que se ha producido declinación de las infecciones como causa de mortalidad. Temas que antes no eran relevantes, se han ido incrementando muy silenciosamente, como la mortalidad por injurias no intencionales y sobre todo las relacionadas con accidentes de tránsito, constituyendo la primera causa de mortalidad entre los 15 y 29 años $^{2}$. Por otra parte está apareciendo con fuerza en sectores adolescentes un aumento de alcoholismo y drogadicción que a menudo se asocia con violencia.

El 66,5\% de los escolares percibe que sus familias son disfuncionales. Casi la mitad de los escolares implicados en este estudio manifiesta que sus padres no dedican el tiempo adecuado para conversar, además, refieren falta de conocimiento de las actividades de sus hijos, falta de reglas claras en la casa, ausencia de rituales familiares y de actividades conjuntas. Todo lo cual podría estar relacionado con el nivel sociocultural de las familias y el cambio que ha sucedido en las familias chilenas, que impiden una conexión adecuada con los hijos, lo que sumado a distorsiones en las representaciones mentales acerca de la tarea educativa del padre y la madre ${ }^{14}$ determinarían la presencia de mayores conductas de riesgo en escolares. Tal como ha descrito Blum ${ }^{11}$, la disponibilidad de tiempo de los padres hacia sus hijos, la presencia de rituales familiares y las actividades compartidas son factores protectores. La presencia de un adecuado vínculo familiar disminuye significativamente la posibilidad de todas las conductas de riesgo en el adolescente, a pesar de la existencia, en el mismo adolescente, de factores de riesgo significativos ${ }^{3,15}$.

Por otra parte, numerosas investigaciones muestran que el consumo de tabaco y alcohol en adolescentes chilenos ha ido en aumento y es cada vez mayor a edades más tempranas ${ }^{16,17}$. En nuestro estudio, las dos sustancias socialmente aceptadas (tabaco y alcohol) son las que registran mayor prevalencia, existiendo diferencias significativas por sexo. En relación al consumo de tabaco, nuestros resultados concuerdan con lo 
encontrado por el CONACE, en su Sexto Estudio Nacional de Drogas en escolares chilenos, donde el consumo es mayor en el sexo femenino. En cambio, el consumo de drogas ilícitas como marihuana y cocaína es mayor en escolares de sexo masculino, lo cual también concuerda con lo encontrado por el CONACE ${ }^{16}$. Por su parte, destaca la alta prevalencia de consumo de tranquilizantes por parte de las mujeres, la cual duplica la de los varones.

Los adolescentes que perciben disfuncionales sus familias presentan mayor riesgo de consumir las cinco sustancias analizadas, sin embargo, este riesgo fue significativo únicamente en el caso del tabaco, marihuana y tranquilizantes. Las restricciones sociales acerca de las sustancias consideradas en este estudio, aumenta su riesgo de consumo en adolescentes que perciben sus familias como disfuncionales, siendo el riesgo menor para el alcohol $(\mathrm{OR}=1,3)$ y el mayor para la cocaína ( $\mathrm{OR}=2,23)$. Además, destaca la influencia de los padres como modelos de consumo de sustancias por parte de sus hijos; de hecho, el consumo de tabaco, alcohol y marihuana por parte del padre o de la madre se asocia significativamente a un mayor consumo por parte de los hijos, destacándose el caso de la marihuana, cuyo consumo es cinco veces mayor en adolescentes cuyo padre la consume y seis veces mayor en el caso de ser consumida por la madre. Estos resultados concuerdan con lo señalado por Blum ${ }^{11}$ en su estudio longitudinal, en el que se comprueba que el ambiente familiar juega un rol importante en adquirir conductas de riesgo o predisponentes para el mismo si existen pobres habilidades interpersonales, baja tolerancia al estrés, baja responsabilidad, evitación de la interacción, problemas de comunicación entre padres e hijos ${ }^{18}$. Por otra parte, la prevalencia de relaciones sexuales (una o más veces) alcanza casi 50\% en varones, siendo significativamente mayor que en mujeres (40\%). El riesgo de mantener relaciones sexuales es significativamente superior en hogares percibidos como disfuncionales, en los cuales la prevalencia para ambos sexos alcanza 50\%. Es alarmante el hallazgo que en el grupo de escolares de 14 a 15 años, $55 \%$ ya ha iniciado actividad sexual, a una edad en que aún no se ha completado el proceso de conformación de su identidad personal, no están psicológicamente maduros aún para establecer una relación de pareja estable y no están capacitados para afrontar las consecuencias de su actividad sexual temprana, como el embarazo, las infecciones de transmisión sexual y las consecuencias emocionales.

La prevalencia de los síntomas emocionales analizados es muy variable, destacando el alto porcentaje de adolescentes de ambos sexos que refieren sentirse inestables e inseguros todo el tiempo (62\% varones y 79,8\% mujeres), seguido del síntoma "sentirse desesperado, descorazonado, sintiendo que nada vale la pena" (19,8\% varones y 25,6\% mujeres). Con excepción de la capacidad de autocontrol, la prevalencia de todos los síntomas emocionales es mayor en mujeres, duplicando e incluso triplicando la prevalencia de los varones (ansiedad y tristeza, respectivamente). El riesgo de presentar los cinco síntomas emocionales descritos es significativamente superior en familias percibidas como disfuncionales, en comparación a familias percibidas como funcionales.

Se encontró una serie de síntomas presentes en escolares chilenos, que pueden corresponder o evolucionar hacia un cuadro depresivo. Llama la atención que $6,1 \%$ de los escolares refiere sentirse "extremadamente" desesperado y sin sentido, lo que indica alto riesgo de suicidio en este grupo. Algunos autores han descrito que la identificación de este grupo de adolescentes a través de cuestionarios de screening realizados en establecimientos escolares es una estrategia efectiva en el diagnóstico precoz y manejo adecuado de la población de adolescentes en riesgo de suicidio ${ }^{19}$. En estudios realizados en Chile, se observan también dificultades de carácter emocional y social, tales como baja autoestima y conductas agresivas que los profesores reconocen en las salas de clase, no obstante no se sienten con la preparación suficiente para trabajar con los estudiantes y con sus familias $^{20}$.

Este estudio cuenta con la limitación del diseño transversal, en que se realiza medición de las variables independientes y dependientes simultáneamente y además, tanto las variables independientes como las conductas evaluadas en los adolescentes resultan de mediciones en los 
mismos individuos, por lo que puede presentar el fenómeno de causalidad reversa, no pudiendo asignarse con claridad el rol de "causa" y "efecto" a una variable.

En conclusión, existe alta prevalencia de síntomas emocionales, percepción de disfunción familiar por parte de los adolescentes participantes en este estudio y conductas de riesgo como consumo de alcohol, drogas y actividad sexual precoz en adolescentes escolares chilenos. La utilización de cuestionarios de screening de síntomas emocionales y conductas de riesgo a nivel escolar, para un diagnóstico precoz e implementación temprana de medidas de prevención y tratamiento a nivel familiar tanto escolar como individual, parece ser una estrategia importante. Todas las conductas de riesgo son más frecuentes en adolescentes provenientes de familias percibidas por ellos como disfuncionales, lo que sugiere la urgencia de promover acciones para fortalecer la familia, un factor protector importante en la prevención de conductas de riesgo en adolescentes. Familia que

\section{REFERENCIAS}

1. Florenzano R. Conductas de riesgo adolescentes y factores protectores. En: Florenzano, Valdés eds. El Adolescente y sus Conductas de Riesgo. Ediciones Universidad Católica de Chile. Tercera Edición 2005; 99-112.

2. Blum R. The health of young people in a global context. J Adolesc Health 2004; 35: 402-18.

3. Resnick M, Bearman P, Blum R. Protecting adolescents from harm. JAMA 1997; 278: 823-32.

4. Borowski I, Ireland M, Resnick M. Adolescent suicide attempts: risks and protectors. Pediatrics 2001; 10: 485-93.

5. Scal P, Ireland M, Borowski I. Tabaquismo en adolescentes americanos. J Community Health 2003; 28: 79-97.

6. Santelices L, Zubarew T, SANTANDER RS. Igualdad de oportunidades, funcionamiento familiar y conductas de riesgo en estudiantes de pedagogía. Boletín de Investigación Educacional 2006; 21,1: 5-6. debe verse apoyada y no cuestionada por el resto de las instituciones y que debe recuperar su papel educativo insustituible en el crecimiento de sus miembros. La intervención no debería centrarse únicamente en los adolescentes, sino debería incorporar un grupo de variables culturales y de calidad de la interacción familiar, que actúan como facilitadores de un desarrollo sano ${ }^{21}$.

Este estudio enfatiza la necesidad inminente de implementar programas y políticas de salud y educación dirigidas a profesionales de salud y educación y la comunidad en general, orientadas al refuerzo de la comunicación y fortalecimiento de lazos en familias con hijos adolescentes.

\section{Agradecimientos}

Agradecemos al Sr. Luis Villarroel, Magíster en Estadística, Departamento de Salud Pública, Pontificia Universidad Católica de Chile, por su valiosa colaboración en la tabulación de los datos y en el análisis estadístico.
7. Santander RS. Salud y rendimiento académico. Factores y conductas de riesgo en alumnos de pedagogía. Colección DIUMCE № 8. Vicerrectoría académica. Universidad Metropolitana de Ciencias de la Educación. 2007.

8. Zubarew T, Laval L. Guía para la supervisión de salud del adolescente. En: Zubarew T, Romero MI, Poblete F, ed. Adolescencia. Promoción, prevención y atención de salud. Ediciones Universidad Católica de Chile. Santiago de Chile. 2003; 85-102.

9. Jessor R. Risk behavior in adolescence: A psychosocial framework for understanding and action. J Adolesc Health 1991; 12: 597-605.

10. VINER R, HaINES M. Variations in associations of health risk behaviors among ethnic minority early adolescents. J Adolesc Health 2006; 28: 55e15$55 \mathrm{e} 23$.

11. Blum R, Halcon L. Adolescent heath in the Caribbean: Risk and Protective Factors. Am J Public Health 2003; 93: 456-60.

12. Florenzano R, Pino P, Marchandón A. Conductas 
de riesgo en escolares de Santiago de Chile. Rev Méd Chile 1993; 121: 462-9.

13. Organización Panamericana de la Salud. Washington D.C. Familia y Adolescencia. Indicadores de Salud. Manual de aplicación de instrumentos e instrumento abreviado. 1999.

14. Santelices L, Pedreros MA. Representaciones cognitivas de padres y estudiantes universitarios acerca de la familia y sus funciones educativas. Revista Paideia 2004; 36: 63-76.

15. Topolski T, Patrick D. Quality of life and healthrisk behaviors among adolescents. J Adolesc Health 2001; 29: 426-35.

16. Sexto Estudio Nacional de Drogas en Población Escolar de Chile, Octavo a Cuarto Medio. CONACE. Gobierno de Chile 2005.
17. Encuesta Mundial de Salud Escolar. Chile 2004. Ministerio de Salud de Chile. OMS. OPS. CDC.

18. Bringottr. La escuela ante los niños maltratados. Editorial Paidos. Buenos Aires. Cuestiones de Educación. 2001.

19. Friedman R. Uncovering an Epidemic - Screening for mental illness in teens. N Engl J Med 2006; 355; 26: 2717-19.

20. Santelices L, Luissi V. Estudio de percepciones de profesores acerca de variables afectivas, sociales y académicas en hijos de padres separados. Boletín de Investigación Educacional 2003; 18: 221-32.

21. Aubert A. Parents, Children and Adolescents. Interactive relationships and development in context. The Haworth Press. New Cork, London. 1997. 